\title{
The question of ambiguity in mathematics and some of arising from these extraordinary consequences. (elementary aspect)
}

\section{REUVEN TINT ${ }^{1}$, PROF. DR. K. RAJA RAMA GANDHI ${ }^{1}$, MICHAEL TINT ${ }^{2}$}

Number Theorist, Israel ${ }^{1}$

Resource person in Math for Oxford University Press and Professor at BITS-Vizag ${ }^{1}$

Software Engineer, Israel $^{2}$

Email: reuven.tint@gmail.com, editor126@gmail.com, tintmisha@gmail.com

http://ferm-tint.blogspot.co.il/

Abstract. This paper proposes a complete elementary without reference to other sources (except for Euler), first proof of the Fermat's Last Theorem. We resolve the question of the ambiguous expansion in the Binomial theorem, Resolved the question of the ambiguity certain infinite sequences of the Euler. Given other versions proof of FLT, We consider and solve the question of not available modern geometric representation of continuous functions without derivatives at any point.

\section{$\S 1$}

1.1. The conventional (classical) expansion in powers

$$
\mathrm{C}_{n}^{n-i} \times x^{i} a^{n-i}
$$

(here, $\mathrm{i}=\mathrm{n}, \mathrm{n}-1, \ldots ., 2,1,0 ; \mathrm{C}_{n}^{i}=\frac{n !}{i !(n-i) !}$;

$a-$ is arbitrary real number;

$\mathrm{n} !=\mathrm{n} \times(\mathrm{n}-1) \times \ldots \times 3 \times 2 \times 1)$

of identities

$$
(\mathrm{x}+a)^{n} \equiv \sum_{i=n}^{i=0} C_{n}^{n-i} x^{i} a^{n-i}[\mathbf{1}] \equiv
$$

$\equiv c_{n}^{0} x^{n}+c_{n}^{1} x^{n-1} a+\ldots+c_{n}^{n-1} x a^{n-1}+c_{n}^{n} a^{n}$ (binomial theorem)

is identically equal to the decomposition

$$
\begin{aligned}
& (\mathrm{x}+a)^{n} \equiv x^{n}+a \times \sum_{j=n-1}^{j=0}(x+a)^{n-1-j} x^{j} \equiv \\
& \equiv x^{n}+a\left[(x+a)^{0} x^{n-1}+(x+a)^{1} x^{n-2}+\right. \\
& \left.+\ldots+(x+a)^{n-2} x+(x+a)^{n-1} \times x^{0}\right][2]
\end{aligned}
$$

[here, $j=(n-1),(n-2), \ldots, 2,1,0]$,

using [2] 


$$
\begin{gathered}
\frac{(x+a)^{n}-x^{n}}{a} \equiv x^{n-1}+(x+a)^{1} x^{n-2}+ \\
+(x+a)^{2} x^{n-3}+\cdots+(x+a)^{n-2} x^{1}+(x+a)^{n-1}-
\end{gathered}
$$

- is the sum of a geometric progression:

1.1.2. with a first member $a_{1}=x^{n-1}$ and common ratio $q=\frac{x+a}{x}$, then

$$
\frac{x^{n-1}\left[\left(\frac{x+a}{x}\right)^{n}-1\right]}{\frac{x+a}{x}-1} \equiv \frac{(x+a)^{n}-x^{n}}{a}[3]
$$

1.1.3. with a first member $a_{1}=(x+a)^{n-1}$ and $q=\frac{x}{x+a}$, then

$$
\frac{(x+a)^{n-1}\left[\left(\frac{x}{x+a}\right)^{n}-1\right]}{\frac{x}{x+a}-1} \equiv \frac{x^{n}-(x+a)^{n}}{-a} \equiv \frac{(x+a)^{n}-x^{n}}{a}[4] \text {. }
$$

1.1.4. This, surprisingly, so far remained unnoticed. Consequences associated with the ambiguity of such an expansion binomial theorem, as will be shown below are very important.

1.1.5. Using [1] and [2] follows, that

$$
\begin{gathered}
c_{n}^{0} x^{n}+c_{n}^{1} a x^{n-1}+c_{n}^{2} a^{2} x^{n-2}+\cdots+ \\
c_{n}^{n-2} a^{n-2} x^{2}+c_{n}^{n-1} a^{n-1} x^{1}+c_{n}^{n} a^{n} x^{0} \equiv \\
\equiv x^{n}+a x^{n-1}+a(x+a)^{1} x^{n-2}+\cdots+ \\
+a(x+a)^{n-3} x^{2}+a(x+a)^{n-2} x^{1}+a(x+a)^{n-1} x^{0} \quad[\mathbf{5}] ;
\end{gathered}
$$

1.1.6. Therefore, coefficients for each $x^{n-i}$ in both sides of the identity[5], except $x^{n}$, respectively, are not equal to each other:

$$
\begin{gathered}
c_{n}^{1} a \neq a ; c_{n}^{2} a^{2} \neq a(x+a) ; \ldots \ldots \ldots \ldots \ldots \\
\ldots \ldots \ldots ; c_{n}^{n-2} a^{n-2} \neq a(x+a)^{n-3} ; \\
c_{n}^{n-1} a^{n-1} \neq a(x+a)^{n-2} ; c_{n}^{n} a^{n} \neq a(x+a)^{n=1}
\end{gathered}
$$

1.1.7. Example.

1)

$$
\begin{gathered}
(2+1)^{5}=2^{5}+5 \times 2^{4} \times 1+\frac{5 \times 4}{1 \times 2} \times 2^{3} \times 1^{2}+\frac{5 \times 4 \times 3}{1 \times 2 \times 3} \times 2^{2} \times 1^{3}+ \\
+\frac{5 \times 4 \times 3 \times 2}{1 \times 2 \times 3 \times 4} \times 2 \times 1^{4}+1^{5}= \\
=2^{5}+(5) \times 2^{4}+\left(10 \times 1^{2}\right) \times 2^{3}+\left(10 \times 1^{3}\right) \times 2^{2}+\left(5 \times 1^{4}\right) \times 2^{1}+ \\
+1^{5} \times 2^{0}=32+80+80+40+10+1=243=3^{5} .
\end{gathered}
$$


2)

$$
\begin{aligned}
(2+1)^{5}=2^{5}+ & (1) \times 2^{4}+[(2+1) \times 1] \times 2^{3}+ \\
+ & {\left[(2+1)^{2} \times 1 \times 2^{2}\right]+\left[(2+1)^{2} \times 1 \times 2^{2}\right]+\left[(2+1)^{3} \times 1\right] \times 2^{1}+} \\
& +(2+1)^{4} \times 2^{0}=32+16+24+36+54+81=243=3^{5} .
\end{aligned}
$$

1.2. With respect to the [1] and [2] we have two geometric progression:

$$
\begin{gathered}
x^{n-1}+x^{n-2}+\cdots+x^{0} \\
(x+a)^{0}+(x+a)^{1}+\cdots+(x+a)^{n-1}
\end{gathered}
$$

The sum of the corresponding multiplication-pairs which is identically equal to

$$
\frac{(x+a)^{n}-x^{n}}{a} \equiv \sum_{i=1}^{n} c_{n}^{i} x^{n-i} a^{i-1} \equiv \sum_{i=1}^{n} x^{n-i}(x+)^{i-1} \quad[6]-
$$

- is hitherto unknown identity.

1.3. For $|x|<1$ and $n \rightarrow \infty$ there polynomials [1] and [2] transformed into power series. Therefore, the statement that the function cannot have two different power series expansions, is wrong in this case, i.e. incorrect in this case uniqueness theorem the power series expansion (proves the method of comparing coefficients).

"If two series

$$
\sum_{n=0}^{\infty} a_{n} x^{n} \text { and } \sum_{n=0}^{\infty} b_{n} x^{n}
$$

are converging in the same range and at all points (or at least in an infinite set of points having zero as a limit point) have the same sums, then series of coincide i.e. $\quad a_{n}=b_{n}$ for $n=$ 0,1,2, ...... “ (IN Bronstein K.A.Semendyaev, "Handbook of Mathematics", Moscow, "Science", Glavfizmatgiz, 1986, page 280.)

\section{Consequence I:}

1.4. Statement. If any multiplications are fragments comprising the binomial theorem, then the expansion of the multiplications on the same variable in a power series is ambiguous (decomposition equal but different), without consideration all versions of the

$\left(x_{i}+a_{i}\right)^{n}=\left(x_{j}+a_{j}\right)^{n}$ for $i \neq j$.

\section{Proof}

In particular, the sequence of Euler

$$
f(x)=(1-x)\left(1-x^{2}\right) \ldots\left(1-x^{n}\right) \equiv(1-x)^{n} F(x)[16]
$$

Since, in the $\S 1$ binomial $(1-x)^{n}$ is ambiguous, we see that and function $f(x)$ is ambiguous. 


\section{$\S 2$}

\section{Consequence II:}

The method of undetermined coefficients.

2.1. With respect to the $\S \mathbf{1}$ we have the ambiguity of the method of undetermined coefficients in the decomposition of proper rational fraction into a sum of partial fractions, if the numerator of this fraction is the binomial statement.

2.1.1. As for the practice is important a final result, We may note that, that this statement is contrary to the fundamental theorem of algebra of rational fractions, which states: "Every proper rational fraction can be decomposed in a unique way into a sum of partial fractions." (AI Kostrikin, "Fundamentals of Algebra", Moscow, Physical and Mathematical Literature, 2000, Theorem 3, pp. 205-206; IN Bronshtein, KA Semendyaev "Handbook of Mathematics", Moscow, "Science", Glavfizmatgiz, 1986, p. 172).

Consider the statement in paragraph.2.1. for example: We have from [1]

\subsection{2.}

$$
\begin{gathered}
\frac{(x-2)^{2}}{(x+1)(x+2)(x+3)}=\frac{x^{2}-4 x+4}{(x+1)(x+2)(x+3)}= \\
=\frac{A}{x+1}+\frac{B}{x+2}+\frac{C}{x+3}= \\
=\frac{x^{2}(A+B+C)+x(5 A+4 B+3 C)+(6 A+3 B+2 C)}{(x+1)(x+2)(x+3)}
\end{gathered}
$$

It follows that,

$$
\left\{\begin{array}{c}
A+B+C=1 \\
5 A+4 B+3 C=-4 \\
6 A+3 B+2 C=4
\end{array}\right.
$$

$A=\frac{9}{2} ; B=-16 ; C=\frac{25}{2} ;$ and

$$
\frac{x^{2}-4 x+4}{(x+1)(x+2)(x+3)}=\frac{\frac{9}{2}}{x+1}+\frac{-16}{x+2}+\frac{\frac{25}{2}}{x+3}
$$

2.1.3. Using [2]

$$
\begin{gathered}
\frac{x^{2}-2 x-2(x-2)}{(x+1)(x+2)(x+3)}= \\
=\frac{x^{2}(A+B+C)+x(5 A+4 B+3 C)+(6 A+3 B+2 C)}{(x+1)(x+2)(x+3)}
\end{gathered}
$$


We obtain,

$$
\left\{\begin{array}{c}
A+B+C=1 \\
5 A+4 B+3 C=-1 \\
6 A+3 B+2 C=-2(x-2)
\end{array},\right.
$$

$A=\frac{7}{2}-x ; B=2 x-12 ; C=\frac{19}{2}-x ;$ and

$$
\frac{x^{2}-2 x-2(x-2)}{(x+1)(x+2)(x+3)}=\frac{\frac{7}{2}-x}{x+1}+\frac{2 x-12}{x+2}+\frac{\frac{19}{2}-x}{x+3} \quad[8]
$$

For $x=1$ :

using

using [8]

$$
\frac{9}{4}-\frac{16}{3}+\frac{25}{8}=\frac{1}{24}
$$

If $x=5$

$$
\frac{5}{4}-\frac{10}{3}+\frac{17}{8}=\frac{1}{24}
$$

$$
\begin{gathered}
x^{2}-4 x+4=1 x^{2}-2 x-2(x-2) ; \\
5^{2}-4 \times 5+4=5^{2}-2 \times 5-2(5-2)= \\
=5^{2}-20+4=5^{2}-10-6=9 ;
\end{gathered}
$$

Using [7]

Using [8]

$$
\begin{gathered}
\frac{3^{2}}{(5+1)(5+2)(5+3)}=\frac{\frac{9}{2}}{5+1}+\frac{-16}{5+2}+\frac{\frac{25}{2}}{5+3}= \\
=\frac{3}{4}-\frac{16}{7}+\frac{25}{16}=\frac{3}{112} ;
\end{gathered}
$$

$$
\frac{3^{2}}{(5+1)(5+2)(5+3)}=-\frac{1}{4}-\frac{2}{7}+\frac{9}{16}=\frac{3}{112}
$$

\section{§3}

\section{Consequence III:}

Arbitrary polynomial in one variable with constant coefficients always correspond (as an option), two classes are equal polynomials with different coefficients for the same variable, i.e. if

$$
f(x)=c_{n} x^{n}+c_{n-1} x^{n-1}+\cdots+c_{1} x+x_{0}[9]
$$

then it is always possible to make

$$
f_{1}(x)=u_{n} x^{n}+u_{n-1} x^{n-1}+u_{n-2} x^{n-2}+\cdots+u_{1} x+u_{0}[\mathbf{1 0}]=
$$




$$
=f_{2}(x)=u_{n} x^{n}+\vartheta_{n-1} x^{n-1}+\vartheta_{n-2} x^{n-2}+\cdots+\vartheta_{1} x+\vartheta_{0}[\mathbf{1 1}]
$$

for arbitrary values of " $x "$, where $u_{i} \neq \vartheta_{i}$.

\section{Proof}

3.1. Since $f(x)$ is the sum of functions $x^{k}(0 \leq k \leq n)$, multiplied by the corresponding coefficients,

3.1.1. We have from [1]:

$$
\begin{gathered}
{[x+(a-x)]^{k} \equiv \sum_{i=k}^{i=0} C_{k}^{k-i}(a-x)^{k-i} x^{i} \equiv} \\
\equiv x^{k}+C_{k}^{1}(a-x) x^{k-1}+C_{k}^{2}(a-x)^{2} x^{k-2}+\cdots \\
\ldots+C_{k}^{k-2}(a-x)^{k-2} x^{2}+C_{k}^{k-1}(a-x)^{k-1} x+(a-x)^{k}
\end{gathered}
$$

3.1.2. We get from $[2]$ :

$$
\begin{gathered}
{[x+(a-x)]^{k} \equiv x^{k}+(a-x) \times \sum_{j=k-1}^{j=0} a^{k-1-j} x^{j} \equiv} \\
\equiv x^{k}+(a-x) x^{k-1}+a(a-x) x^{k-2}+a^{k-3}(a-x) x^{2}+ \\
+a^{k-2}(a-x) x+a^{k-1}(a-x)[\mathbf{1 3}]
\end{gathered}
$$

3.1.3. Taking in the [9], in particular, without affecting the generality, $n=4$, using [11] we obtain:

$$
\begin{gathered}
f_{1}(x)=c_{4}\left[x^{4}+4(a-x) x^{3}+6(a-x)^{2} x^{2}+4(a-x)^{3} x+\right. \\
\left.+(a-x)^{4}\right]+ \\
+c_{3}\left[x^{3}+3(a-x) x^{2}+3(a-x) x+(a-x)^{3}\right]+ \\
+c_{2}\left[x^{2}+2(a-x) x+(a-x)^{2}\right]+ \\
+c_{1}[x+(a-x)]+ \\
+c_{0}[1]= \\
=c_{4} x^{4}+\left[4 c_{4}(a-x)+c_{3}\right] x^{3}+\left[6 c_{4}(a-x)^{2}+\right. \\
\left.+3 c_{3}(a-x)+c_{2}\right] x^{2}+\left[4 c_{4}(a-x)^{3}+3 c_{3}(a-x)^{2}+\right. \\
\left.+2 c_{2}(a-x)+c_{1}\right] x+\left[c_{4}(a-x)^{4}+c_{3}(a-x)^{3}+\right. \\
\left.+c_{2}(a-x)^{2}+c_{1}(a-x)\right]+c_{0}[\mathbf{1 4}]
\end{gathered}
$$

3.1.4. Now, by [14] the result is:

$$
\begin{gathered}
f_{2}(x)=c_{4}\left[x^{4}+(a-x) x^{3}+a(a-x) x^{2}+a^{2}(a-x) x+\right. \\
\left.+a^{3}(a-x)\right]+
\end{gathered}
$$




$$
\begin{gathered}
+c_{3}\left[x^{3}+(a-x) x^{2}+a(a-x) x+a^{2}(a-x)\right]+ \\
+c_{2}\left[x^{2}+(a-x) x+a(a-x)+\right. \\
+c_{1}[x+(a-x)]+ \\
c_{0}[1]= \\
=c_{4} x^{4}+\left[c_{4}(a-x)+c_{3}\right] x^{3}+\left[c_{4} a(a-x)+c_{3}(a-x)+c_{2}\right] x^{2}+ \\
+\left[c_{4} a^{2}(a-x)+c_{3} a(a-x)+c_{2}(a-x)+c_{1}\right] x+ \\
+\left[c_{4} a^{3}(a-x)+c_{3} a^{2}(a-x)+c_{2} a(a-x)+c_{1}(a-x)\right]+c_{0}
\end{gathered}
$$

3.1.5. Let $c_{4}=2 ; c_{3}=3 ; c_{2}=5 ; c_{1}=7 ; c_{0}=11 ; a=5 ; x=3$.

3.1.5.1. Thus we get from $[14]$ :

$$
\begin{gathered}
f_{1}(3)=2 \times 3^{4}+(4 \times 2 \times 2+3) \times 3^{3}+ \\
+\left(6 \times 2 \times 2^{2}+3 \times 3 \times 2+5\right) \times 3^{2}+ \\
+\left(4 \times 2 \times 2^{3}+3 \times 3 \times 2^{2}+2 \times 5 \times 2+7\right) \times 3+ \\
+\left(2 \times 2^{4}+3 \times 2^{3}+5 \times 2^{2}+7 \times 2\right]+11= \\
=1796=2 \times 3^{4}+19 \times 3^{3}+71 \times 3^{2}+127 \times 3+101
\end{gathered}
$$

3.1.5.2. using $[\mathbf{1 5}]$ :

$$
\begin{gathered}
f_{2}(3)=2 \times 3^{4}+(2 \times 2+3) 3^{3}+(2 \times 5 \times 2+3 \times 2+5) \times 3^{2}+ \\
+\left(2 \times 5^{2} \times 2+3 \times 5 \times 2+5 \times 2+7\right) \times 3+ \\
+\left(2 \times 5^{3} \times 2+3 \times 5^{2} \times 2+5 \times 5 \times 2+7 \times 2\right)+11=1796= \\
=2 \times 3^{4}+7 \times 3^{3}+31 \times 3^{2}+147 \times 3+725,
\end{gathered}
$$

3.1.6. or

$$
\begin{aligned}
& F_{1}(3)=19 \times 3^{3}+71 \times 3^{2}+127 \times 3+90=1623 \\
& F_{2}(3)=7 \times 3^{3}+31 \times 3^{2}+147 \times 3+714=1623
\end{aligned}
$$

this completes the proof.

3.1.7. The same type of duality holds for the derivatives of the Taylor formula.

\section{$\S 4$}

\section{Consequence IV.}

4.1. In the memoir of Euler " Opening of the most extraordinary law of numbers related to the amounts of their divisors ", is given in the book by A. Polya" Mathematics and plausible reasoning "," Science ", Glavfizmatgiz, Moscow, 1975, to claim 13, p.

120 ,

or Euler, "Opera Omnia", ser.1,vol2,p.241-253, claimed that "If the sum of an infinite series equal to zero, then what value of any " $x$ ", the coefficient of each power " $x$ " must necessarily be equal to zero".

As shown below, this statement is

1) is not completely accurate,

2) although absolutely true. 
4.2. Consider the equation

$$
A^{n}+B^{n}=D^{n}[17]
$$

4.2.1. under the following conditions [18]:

1) $A=x+a ; B=x+b ; D=x+c[19]$,

2) $x, A, B-$ are arbitrary real numbers:

$-\infty<x<\infty ;-\infty<A<\infty ;-\infty<B<\infty$;

3) $D-A-B=\sqrt[i]{A^{i}+B^{i}}-A-B$,

$1 \leq i \leq n, \quad n=1,2,3, \ldots \ldots$

4) for $i$ odd

$$
\sqrt[i]{\left(A^{i}+B^{i}\right)<0}
$$

may be less than zero;

5) $a, b, c-$ are corresponding [19] the real numbers

(when $A, B, D$ fixed).

4.2.2. Using [2]

$$
\begin{aligned}
&(x+a)^{n} \equiv x^{n}+a\left(x^{n-1}+A x^{n-2}+\cdots+A^{n-1}\right)[\mathbf{2 0}] \\
&(x+b)^{n} \equiv x^{n}+b\left(x^{n-1}+B x^{n-2}+\cdots+B^{n-1}\right)[\mathbf{2 1}] \\
&(x+c)^{n} \equiv x^{n}+c\left(x^{n-1}+D x^{n-2}+\cdots+D^{n-1}\right)[\mathbf{2 2}]
\end{aligned}
$$

Since, if [17] is true, then

$$
\begin{gathered}
-\{[\mathbf{2 2}]-[\mathbf{2 0}]-[\mathbf{2 1}]\} \equiv \\
\equiv x^{n}-x^{n-1}(c-a-b)-x^{n-2}(c D-a A-b B)- \\
-x^{n-3}\left(c D^{2}-a A^{2}-b B^{2}\right)- \\
-\cdots x\left(c D^{n-2}-a A^{n-2}-b B^{n-2}\right)- \\
-x^{0}\left(c D^{n-1}-a A^{n-1}-b B^{n-1}\right) \equiv 0[\mathbf{2 3}]
\end{gathered}
$$

where the coefficients of each degree " $x$ " not equal to zero (which is defined below).

Consider this, without affecting the community, with examples for $n=3$ and $n=2$.

For $n=3$ from [23]:

$$
\begin{gathered}
x^{3}-x^{2}(c-a-b)-x(c D-a A-b B)-x^{0}\left(c D^{2}-a A^{2}-b B^{2}\right) \equiv \\
\equiv x^{3}-x^{2}(D-x-A+x-B+x)-x[(D-x) D- \\
-(A-x) A-(B-x) B]- \\
-x^{0} \times\left[(D-x) D^{2}-(A-x) A^{2}-(B-x) B^{2}\right] \equiv \\
\equiv x^{3}-\left(x^{2} D-x^{2} A+x^{3}-x^{2} B\right)- \\
-\left(x D^{2}-x^{2} D-x A^{2}+x^{2} A-x B^{2}+x^{2} B\right)- \\
-\left(D^{3}-x D^{2}-A^{3}+x A^{2}-B^{3}+x B^{2}\right) \equiv 0[\mathbf{2 4}] .
\end{gathered}
$$

In the [24] all the coefficients of the power " $x$ " not equal to zero. But after redistribution terms in the $[24]$ become equal to zero:

$$
\begin{gathered}
{[24] \equiv x^{3}(1-1)-x^{2}[(D-D)-(A-A)-(B-B)]-} \\
-x\left[\left(D^{2}-D^{2}\right)-\left(A^{2}-A^{2}\right)-\left(B^{2}-B^{2}\right)\right]- \\
-x^{0}\left(D^{3}-A^{3}-B^{3}\right) \equiv 0[\mathbf{2 5}] .
\end{gathered}
$$


4.3. For $n=2$ from $[23]$ :

4.3.1.

$$
\begin{gathered}
x^{2}-x(c-a-b)-x^{0}(c D-a A-b B)[\mathbf{2 6}] \equiv \\
\equiv x^{2}-x(D-x-A+x-B+x)- \\
-x^{0}[(D-x) D-(A-x) A-(B-x) B] \equiv \\
\equiv x^{2}-\left(x D-x A-x B+x^{2}\right)- \\
-\left(D^{2}-x D-A^{2}+x A-B^{2}+x B\right) \equiv \\
\equiv x^{2} \times(1-1)-x[(D-D)-(A-A)-(B-B)]- \\
-x^{0}\left(D^{2}-A^{2}-B^{2}\right) \equiv 0[27] .
\end{gathered}
$$

In the [26] all the coefficients of the powers " $x$ " not equal to zero. But after redistribution terms in the [27] become equal to zero.

\subsection{2.}

Using [26]

if

$$
(D-A-B+x)^{2}+(D-x)^{2}-(A-x)^{2}-(B-x)^{2} \equiv N^{2}[\mathbf{2 8}],
$$

$x-$ is arbitrary number.

$$
D=p^{2}+q^{2} ; A=p^{2}-q^{2} ; B=2 p q ; N=2 q(p-q) ;
$$

For

For

$$
x=D, \quad(2 D-A-B)^{2} \equiv N^{2}+(A-D)^{2}+(B-D)^{2}[29] .
$$

$$
x=A, \quad(D-A)^{2}+(D-B)^{2} \equiv(A-B)^{2}+N^{2}[\mathbf{3 0}] .
$$

4.4. Generalizing to an arbitrary " $n "$, we argue that in the [23]:

1) the coefficients of each power " $x$ " not equal to zero when

$$
\sum_{i=1}^{n} x^{n-i}\left[\left(D^{i}-A^{i}-B^{i}\right)=\left(\sqrt[i]{A^{i}+B^{i}}\right)^{i}-A^{i}-B^{i}\right] \equiv 0[31]
$$

under conditions of the [18];

2) the coefficients of each power of " $x$ " after the corresponding redistribution terms and under the same conditions of [18] identically equal to zero.

3 ) that is, that if the sum of an infinite series is zero, whatever the value " $x "$, , the coefficient of each power " $x$ " can be either zero or non-zero at the same time for the case considered in the 4.2. .

\section{$\S 5$}

\section{Consequence V.}

\section{The first version of proof for Fermat's Last Theorem.}

"The equation

$$
A^{n}+B^{n}=D^{n}
$$

for $n>2$ has no solutions in integers". 


\section{Proof}

5.1. From [23] it follows that, if

where

$$
D_{j}^{i}-A_{j}^{i}-B_{j}^{i} \equiv 0
$$

$$
1 \leq i \leq n
$$

for each fixed value

$$
1 \leq j \leq m
$$

$\mathrm{m}, \mathrm{n}$-are arbitrary natural numbers, including 1 and 0 , [ (for example, for $D$ (same for $A$ and $B)$ )

$$
\left\{\begin{array}{c}
D_{1}^{1}, D_{1}^{2}, D_{1}^{3}, \ldots, D_{1}^{n} \\
D_{2}^{1}, D_{2}^{2}, D_{2}^{3}, \ldots, D_{2}^{n} \\
\ldots \ldots \ldots \ldots \ldots \ldots . . \\
D_{m}^{1}, D_{m}^{2}, D_{m}^{3}, \ldots, D_{m}^{n}
\end{array}\right.
$$

cover the entire range of possible solutions of the equations [32] for fixed from 1 to $n$ for each $j$ ]

$$
\begin{gathered}
D_{j}=x+c ; A_{j}=x+a ; B_{j}=x+b \\
-\infty<x<\infty
\end{gathered}
$$

,then

$$
x^{n}(1-1)-\sum_{i=1}^{n} x^{n-i} \times \sum_{\substack{i=1 \\ j=1}}^{i=n, j=m}\left(D_{j}^{i}-A_{j}^{i}-B_{j}^{i}\right) \equiv 0
$$

and

$$
\begin{gathered}
x^{n}(1-1)-x^{n-1}\left(D_{j}^{1}-A_{j}^{1}-B_{j}^{1}\right)- \\
-x^{n-2}\left\{\left[\left(p_{j}^{2}+q_{j}^{2}\right) t\right]^{2}-\left[\left(p_{j}^{2}-q_{j}^{2}\right) t\right]^{2}-\left(2 p_{j} q_{j} t\right)^{2}\right\}- \\
-x^{n-3}\left(D_{j}^{3}-A_{j}^{3}-B_{j}^{3}\right)-\cdots-x^{0}\left(D_{j}^{n}-A_{j}^{n}-B_{j}^{n}\right) \equiv 0[\mathbf{3 2}] .
\end{gathered}
$$

Hence, if we require that $D_{j}, A_{j}, B_{j}, p_{j}, q_{j}, t, x$ there were only natural (integer) numbers, then

and

$$
D_{j}^{1}=A_{j}^{1}+B_{j}^{1}
$$

$$
D_{j}^{2}=A_{j}^{2}+B_{j}^{2}
$$

for arbitrary $j$ always have a solution in positive (integer) numbers and [32] gives all solutions. As has been proved yet L. Euler in 1768, the equation

$$
A_{j}^{3}+B_{j}^{3}=D_{j}^{3}
$$

not for any $j$ (in another, generally in integers) has no solutions. 
(MM Postnikov, "Fermat's theorem", Science, Glavfizmatgiz, Moscow, 1978 pp. 31-49) and, it is clear that, the equation

$$
A^{n}+B^{n}=D^{n}
$$

also has no solutions in integers (natural) numbers for $n>2$

5.1.1. Thus, the equation

$$
A^{n}+B^{n}=D^{n}
$$

has solutions in positive integers, if the following condition hold (necessary and sufficient condition), Without loss of generality it can be assumed that in the [32] the whole set of natural (integers) and each triptych (group of terms of three, united by one idea)- are coefficients for $x^{n-i}$ - of the entire length of sequentially changing the exponent " $n$ ", beginning from " $n$ " to 0 , necessarily identically equal to zero. This completes the proof.

5.1.2. Since 5.1.1. we obtain, that unsolvable equations

$$
A^{3}+B^{3}=D^{3}
$$

in integers is a necessary and sufficient condition (is said to be the for $n=1$ and $n=2$ the equation is solvable) unsolvable equations

$$
A^{n}+B^{n}=D^{n}
$$

in integers for $n>3$.

5.1.3. The above proof - is essentially, the algorithm is the establishment of the solvability (suitably modified) many types of Diophantine equations.

\section{$\S 6$}

The second version of proof for Fermat's Last Theorem.

From the obtained fantastic identity [28] we have the identity

$$
(D-A-B)^{2}+D^{2}-A^{2}-B^{2} \equiv N^{2}
$$

,if

$$
D=p^{2}+q^{2} ; A=p^{2}-q^{2} ; B=2 p q ;
$$

$p, q$ - are arbitrary natural numbers, and

$$
N=D-A-B=2 q(p-q) .
$$


Then, if

$$
D^{2 n_{1}}-A^{2 n_{1}}-B^{2 n_{1}}=0
$$

for all natural $n_{1} \geq 1$,it follows that

$$
\left(D^{n_{1}}-A^{n_{1}}-B^{n_{1}}\right)^{2}+\left(D^{n_{1}}\right)^{2}-\left(A^{n_{1}}\right)^{2}-\left(B^{n_{1}}\right)^{2}=N^{2} .
$$

But

$$
D^{n_{1}}-A^{n_{1}}-B^{n_{1}}=2 q(p-q) \neq 0
$$

not for any natural $\quad n_{1} \geq 1$. we obviously have, for natural $n>2$ the equation

$$
A^{n}+B^{n}=D^{n}
$$

has no solutions in positive integers. This completes the proof.

\section{$\S 7$}

\section{The third version of proof for Fermat's Last Theorem.}

Proof is based on the (6), proceeding, in particular, from the obtained by us the following identity:: $\left[\mathrm{x}\left(3 y^{2}-x^{2}\right)\right]^{2}+\left[y\left(3 x^{2}-y^{2}\right)\right]^{2} \equiv\left(z^{3}\right)^{2}$,

if

$$
x^{2}+y^{2}=z^{2}
$$

\section{Proof}

1. We obtain follows identity:

$$
x(3 y-x)^{2}+y(3 x-y)^{2} \equiv z^{3}[33]
$$

if

$$
x+y=z
$$

1.1. With respect to [33]

$$
3 x y z \equiv z^{3}-x^{3}-y^{3}[34] .
$$

This means that,

$$
x^{3}+y^{3} \neq z^{3}[35]
$$

since, none of the integers $x, y, z$ in the [34] cannot be equal to 0 ,i.e. equation [35] has no solutions in integers. This completes the proof of FLT.

1.2. Let

$$
x^{n}+y^{n}=z^{n}[36]
$$

where $n-$ is arbitrary natural number. 
1.2.1. Combining [34] and [36]

$$
3 x^{n} y^{n} z^{n} \equiv\left(z^{n}\right)^{3}-\left(x^{n}\right)^{3}-\left(y^{n}\right)^{3} \not \equiv 0
$$

has no solutions in integers for $n \geq 3$, as the [35] has no solutions in integers. The proof is complete. This is probably "a truly wonderful proof", that gave Pierre de Fermat.

\section{Examples.}

a) If

$$
x^{2}+y^{2}=z^{2}
$$

,then

$$
3 x^{2} y^{2} z^{2} \equiv z^{6}-x^{6}-y^{6}
$$

Let $x=3 ; y=4 ; z=5$. Therefore,

$$
3 \times 3^{2} \times 4^{2} \times 5^{2}=5^{6}-3^{6}-4^{6}
$$

and

$$
10800=15625-729-4096
$$

b) If

$$
x+y=z
$$

,then

$$
3 x y z=z^{3}-x^{3}-y^{3} .
$$

Let

$$
x=2 ; y=3 ; z=5 \text {. }
$$

Hence,

$$
3 \times 2 \times 3 \times 5=5^{3}-2^{3}-3^{3}
$$

and

$$
90=125-8-27
$$

\section{Remarks:}

1. $x$ and $y$ are completely arbitrary real numbers. And this fact is expressed by the formula (identity)

$$
\left(x=x_{1}^{n}\right)+\left(y=y_{1}^{n}\right) \equiv(\sqrt[n]{z})^{n}
$$

for arbitrary real $x_{1}$ and $y_{1}$ and for arbitrary positive integers $n \geq 1$, where may sometimes be for $x_{1}, y_{1}$-is an integer $z_{1}=\sqrt[n]{z}$ is an integer $(n=1 ; n=2)$, i.e.

$$
x_{1}^{n}+y_{1}^{n}=z_{1}^{n} \text {. }
$$


For $n=1$

$$
\left(x_{1}=x\right)+\left(y_{1}=y\right) \equiv\left(z_{1}=z\right)
$$

2. Multiplying both sides of the Pythagorean equation

by

$$
x^{2}+y^{2}=z^{2}
$$

$$
9\left(x^{4}+y^{4}+z^{4}\right)
$$

,we get

$$
\begin{gathered}
y^{2}\left[9\left(x^{4}+y^{4}+z^{4}\right)+x^{2}\left[9\left(x^{4}+y^{4}+z^{4}\right)\right] \equiv\right. \\
\equiv z^{2}\left[9\left(x^{4}+y^{4}+z^{4}\right)\right][\boldsymbol{I}]
\end{gathered}
$$

Adding term by term, we obtain the following equation

$$
\begin{aligned}
& \left(x x^{2}\right)^{2}+\left[y\left(3 z^{2}+y^{2}\right)\right]^{2} \equiv\left[z\left(3 y^{2}+z^{2}\right)\right]^{2}[\boldsymbol{I I}] \\
& {\left[x\left(3 z^{2}+x^{2}\right)\right]^{2}+\left(y y^{2}\right)^{2} \equiv\left[z\left(3 x^{2}+z^{2}\right)\right]^{2}[\boldsymbol{I I I}]} \\
& {\left[x\left(3 y^{2}-x^{2}\right)\right]^{2}+\left[y\left(3 x^{2}-y^{2}\right)\right]^{2} \equiv\left(z z^{2}\right)^{2}[\boldsymbol{I} \boldsymbol{V}],}
\end{aligned}
$$

we obtain

$$
[I I]+[I I I]+[I V] \equiv[I]
$$

\section{§8}

The question of continuous functions, that is not available to our (modern mathematics) geometric concepts.

1. Continuous function

$$
\begin{gathered}
y \equiv \pm 2 q(p-q) \equiv \\
\equiv \pm \sqrt{(D-A-B+x)^{2}+(D-x)^{2}-(A-x)^{2}-(B-x)^{2}}
\end{gathered}
$$

for fixed values of arbitrary numbers

$$
\begin{gathered}
p \neq q, D=p^{2}+q^{2}, A=p^{2}-q^{2}, \\
B=2 p q, \quad y= \pm 2 q(p-q),-\infty<x<\infty
\end{gathered}
$$

(the function [37] is continuous at each point of the interval) has no derivative of any one value $" x "\left(y^{\prime}=0\right)$.

The curve corresponding to this function, which until now has been available to our (modern mathematics) geometric concepts, is a line

$$
y \equiv \pm 2 q(p-q)
$$


, parallel to the axis " $x "$, which countless. For $p=q$ the axis " $x$ " becomes this line, i.e., the entire plane becomes a surface required form.

2. In order to determine the cause of the above, it is necessary [37] shall be taken squared and expand the brackets - " $x "$ will disappear. (associated with the ambiguity in mathematics).

\section{References:}

[1] И.Н. Бронштейн, К.А.Семендяев, “Справочник по математике”, Москва “Наука”, Главфизматгиз, 1986.

[2] А.И. Кострикин, “Основы алгебры”, Москва ,физико-математическая литература, 2000.

[3] А. Пойа "Математика и правдоподобные рассуждения", “Наука", Главфизматгиз, Москва, 1975.

[4] Leonhardi Euleri opera omnia / Ser.1, Opera mathematica. Vol.2, 1915.

[5] М.М. Постников, ” Теорема Ферма”, Наука ,Главфизматгиз, Москва, 1978.

[6] The methods of solving equations with co-prime equal the two only in one of the three possible cases. The proof of Catalan's Conjecture.

a. PROF. DR. K. RAJA RAMA GANDHI, REUVEN TINT, MICHAEL TINT

[7] В.И. Смирнов, “ Курс высшей математики”, т.1 , “Наука”, Главфизматгиз, Москва, 1967, стр. 106. 